\title{
Recent developments of the 1 MV AMS facility at the Centro Nacional de Aceleradores
}

G. Scognamiglio, E. Chamizo, J.M. López-Gutiérrez, A.M. Müller, S.

Padilla, F.J. Santos, M. López-Lora, C. Vivo, M. García-León

International Conference on Accelerator Optimization 7-9 October 2015, Seville 


\section{Outline}

- The facility

- Recent developments

- Results

- Stripper

- Detector

- Conclusions

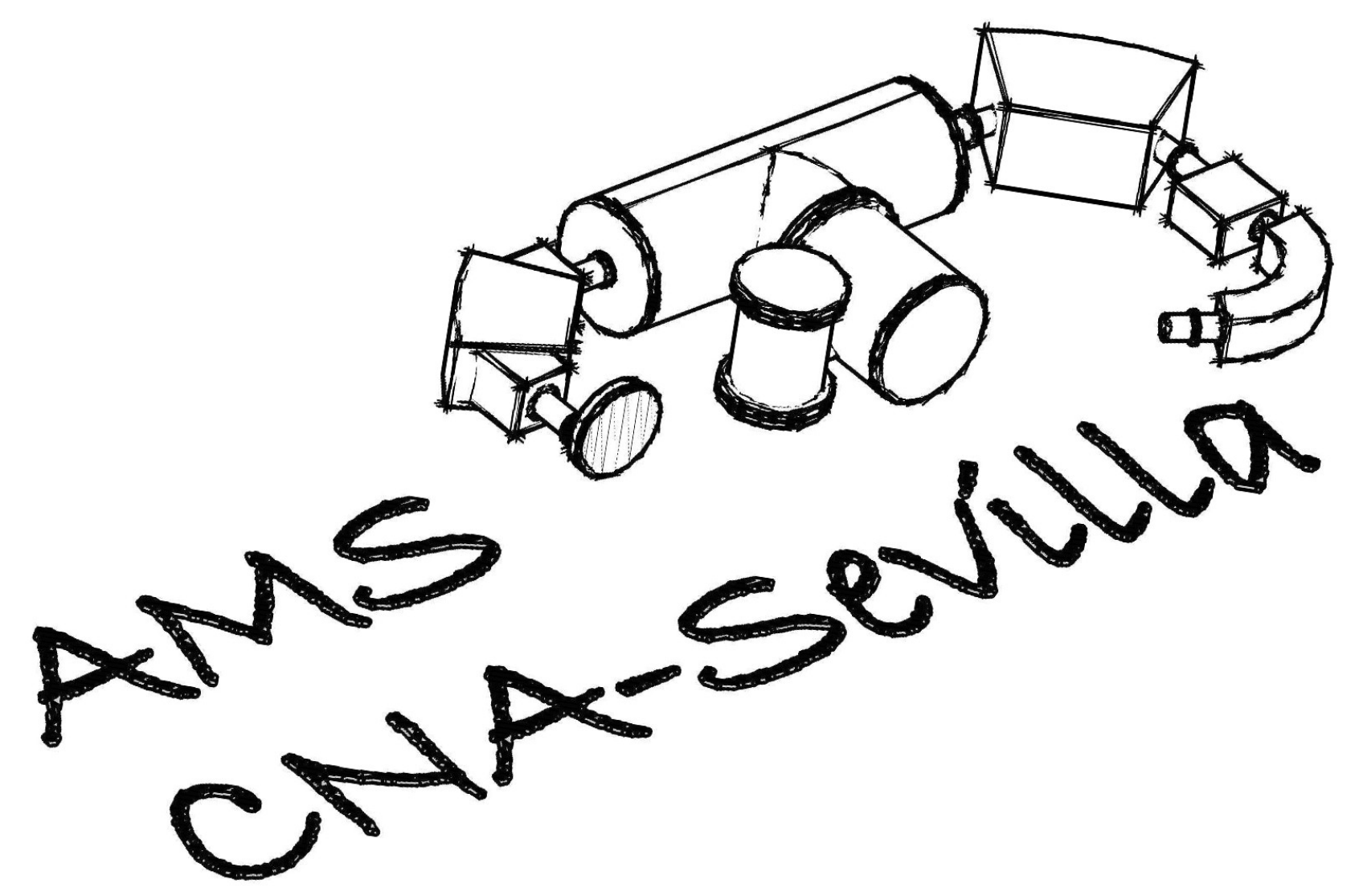




\section{AMS at CNA}

2005

multielemental SARA

(High Voltage Engineering Europe)

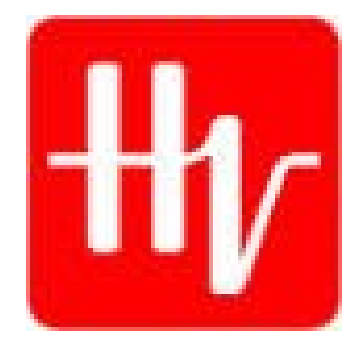

\section{2}

${ }^{14} \mathrm{C}$-dedicated Spanish MICADAS (ETH-Zürich)

ETHzürich lonplus ${ }^{3}$ engineering scientific instruments

Measured isotopes: ${ }^{14} \mathrm{C},{ }^{10} \mathrm{Be},{ }^{26} \mathrm{Al},{ }^{41} \mathrm{Ca},{ }^{129} \mathrm{I},{ }^{236} \mathrm{U}$ and ${ }^{239,240} \mathrm{Pu}$.

- archaeology

- geology

- environmental sciences

- medicine 


\section{SARA: Spanish Accelerator for Radionuclides Analysis}

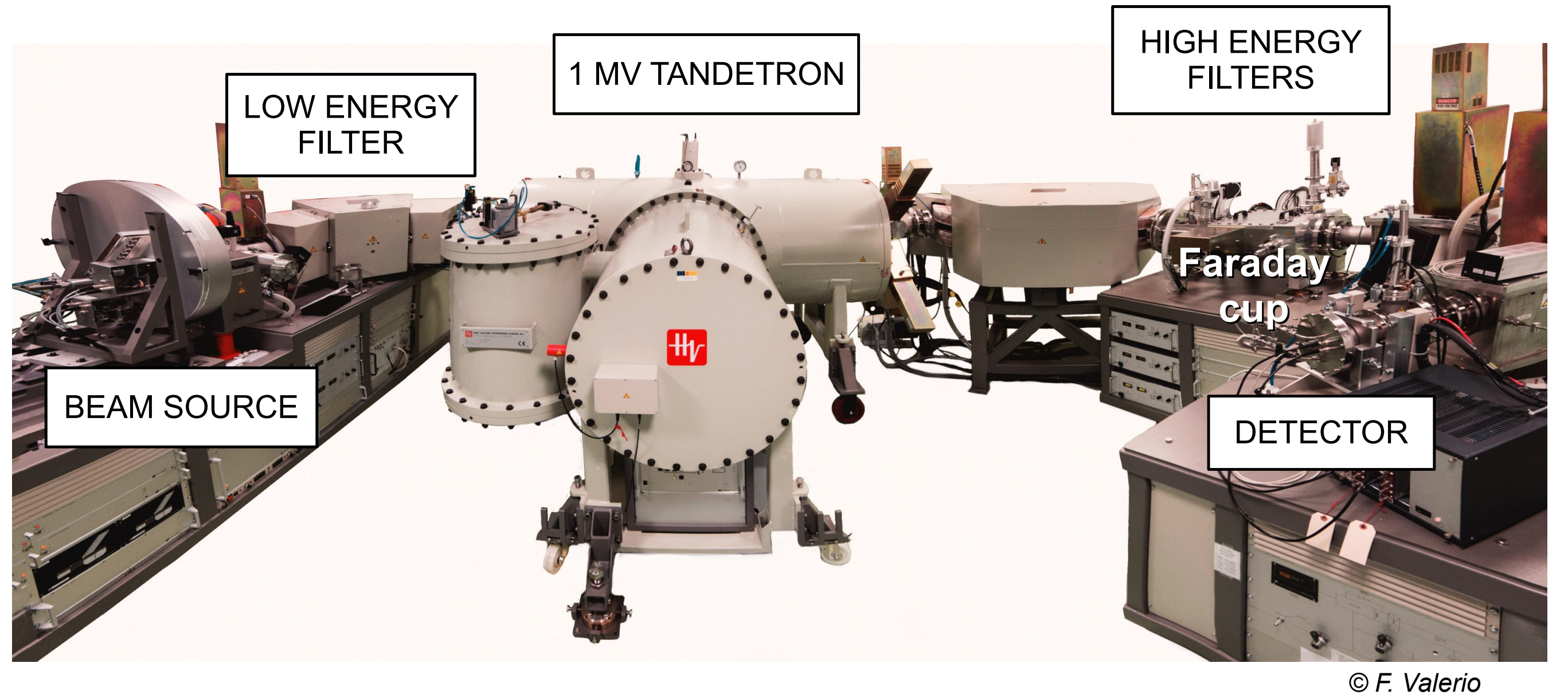




\title{
Recent developments
}

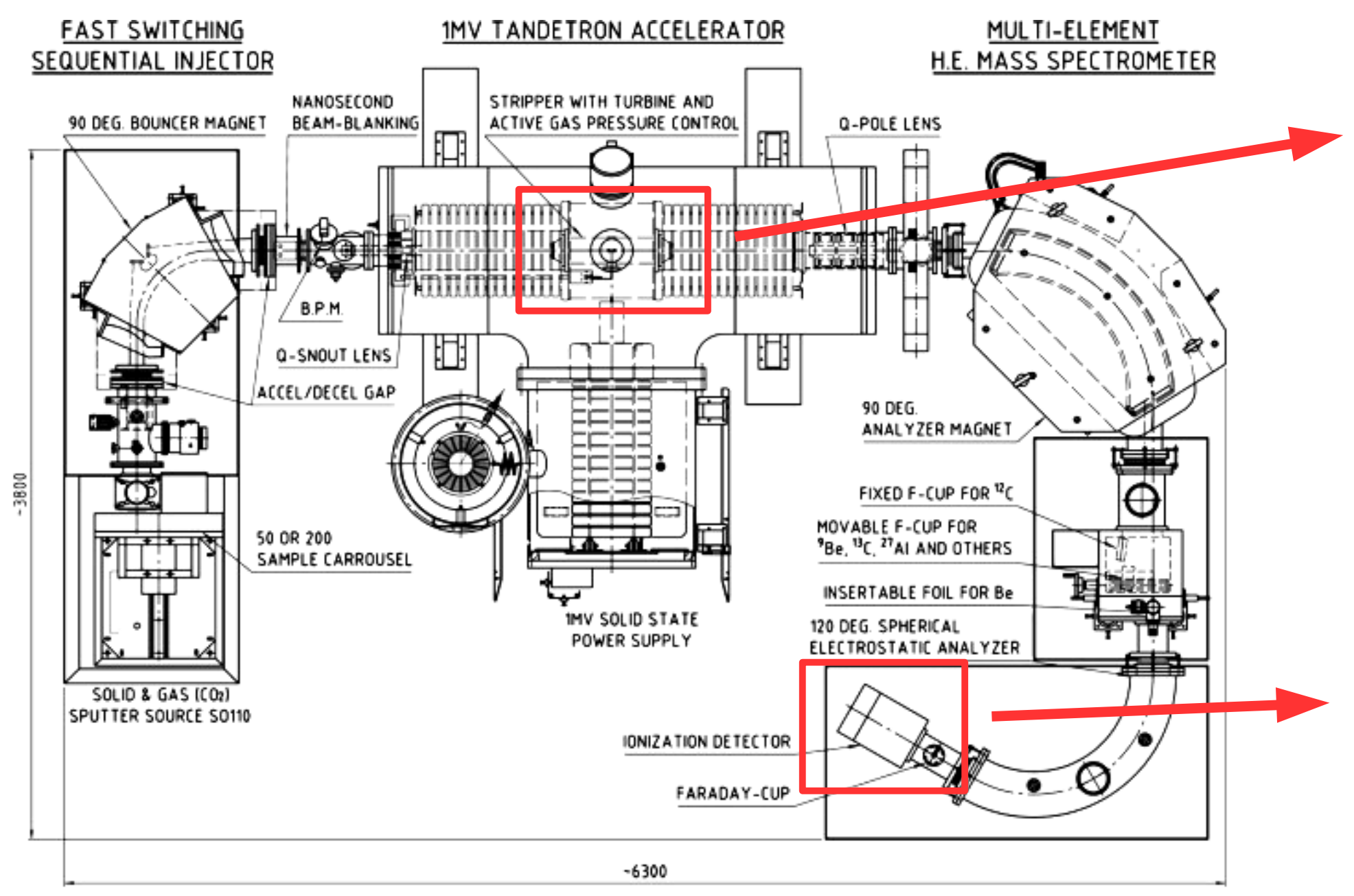

\author{
Helium \\ instead of \\ Argon
}

\section{Low-noise} detector 


\section{Improving the transmission}

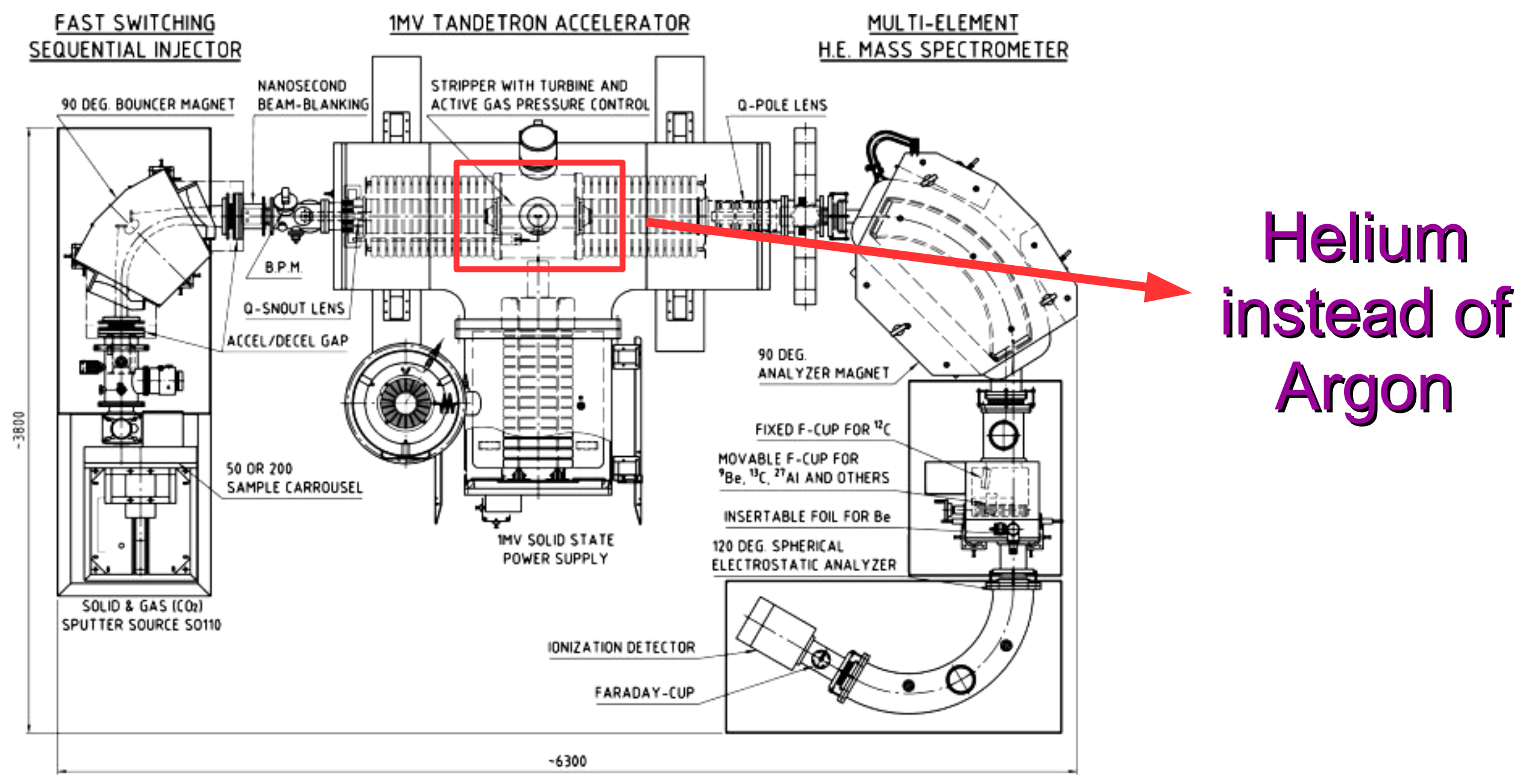




\section{The stripping process}

\section{MV Tandetron}

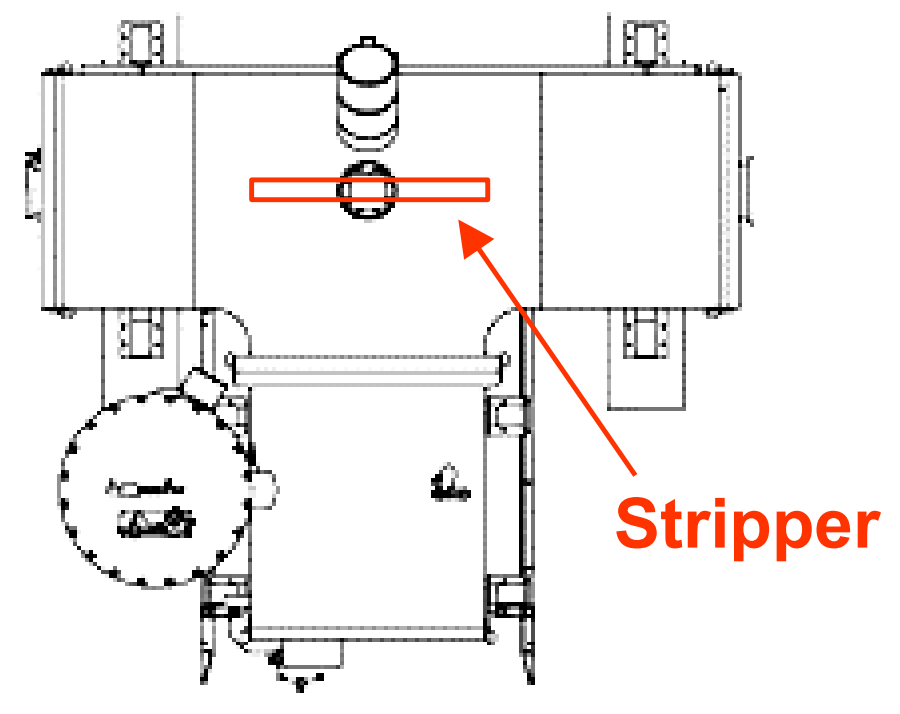

The stripping process:

- Makes the molecules break up
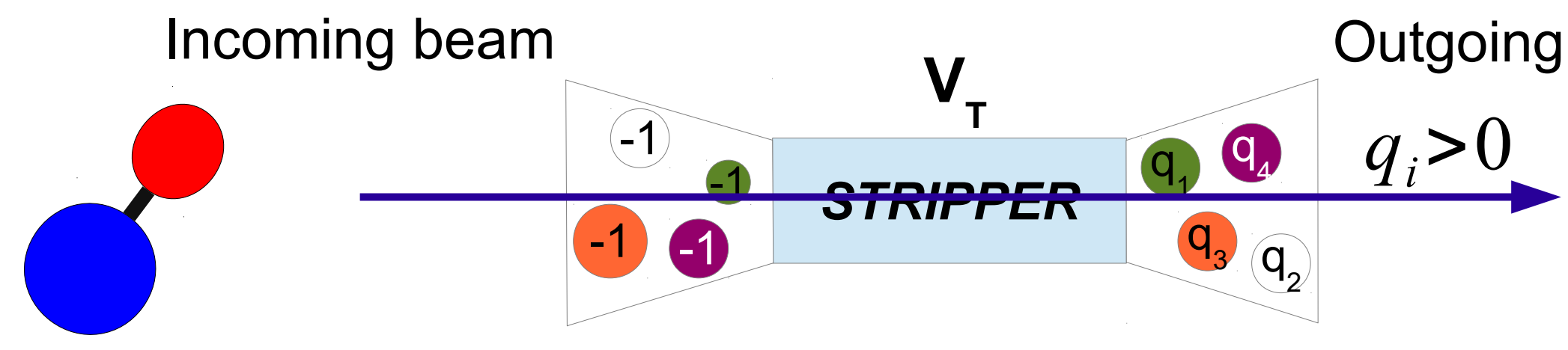

$$
E_{\text {in }}=E_{\text {source }} \quad E_{\text {out }}=\left(\frac{m_{\text {out }}}{M_{\text {in }}}+q\right) e V_{T}+\frac{m_{\text {out }}}{M_{\text {in }}} E_{\text {in }}
$$




\section{The transmission}

$$
\text { Transmission }=\frac{I_{H E}}{q \cdot I_{\mathrm{LE}}}
$$

The transmission for a selected

state charge depends on:

- Nature of the incoming beam,

- Stripping gas,

- Stripper pressure,

- Beam energy.

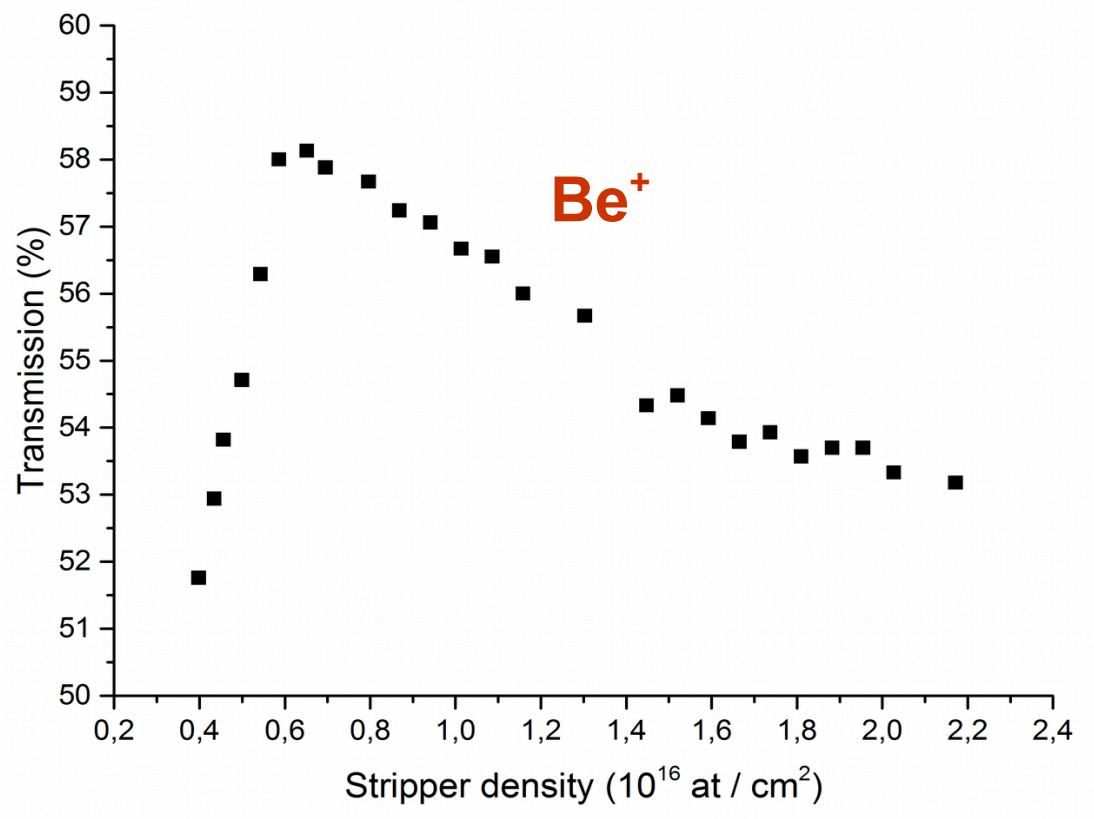

High charge state fractions and minimal scattering losses are essential for low energy AMS 


\section{He as a stripper}

$0.2 \mathrm{MV}$

$0.6 \mathrm{MV}$

$1 \mathrm{MV}$

$3 \mathrm{MV}$

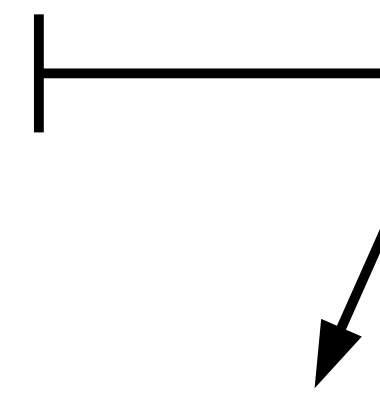

- High charge state yield

- Low scattering losses

Schulze-König et al., 2011 Vockenhuber et al., 2013

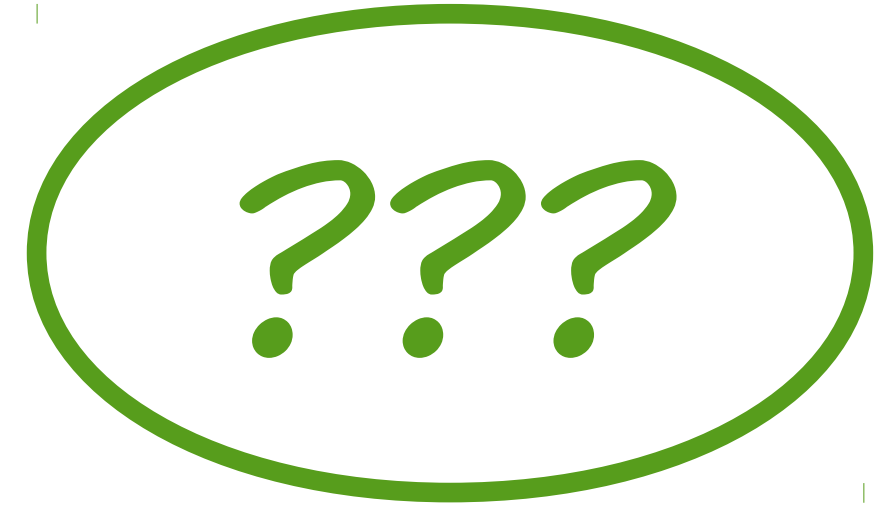

- High charge state yield

Winkler et al., 2015

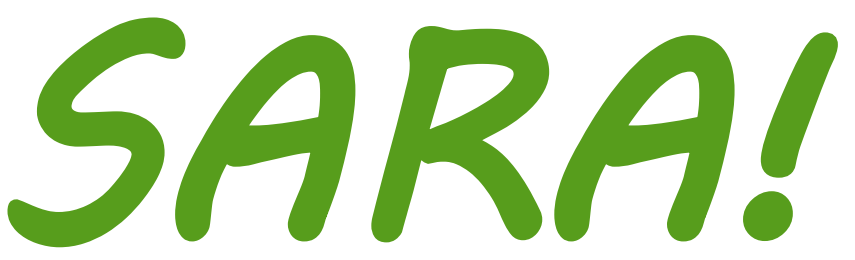




\section{Results}

- Part 1: He stripping

- Part 2: detector

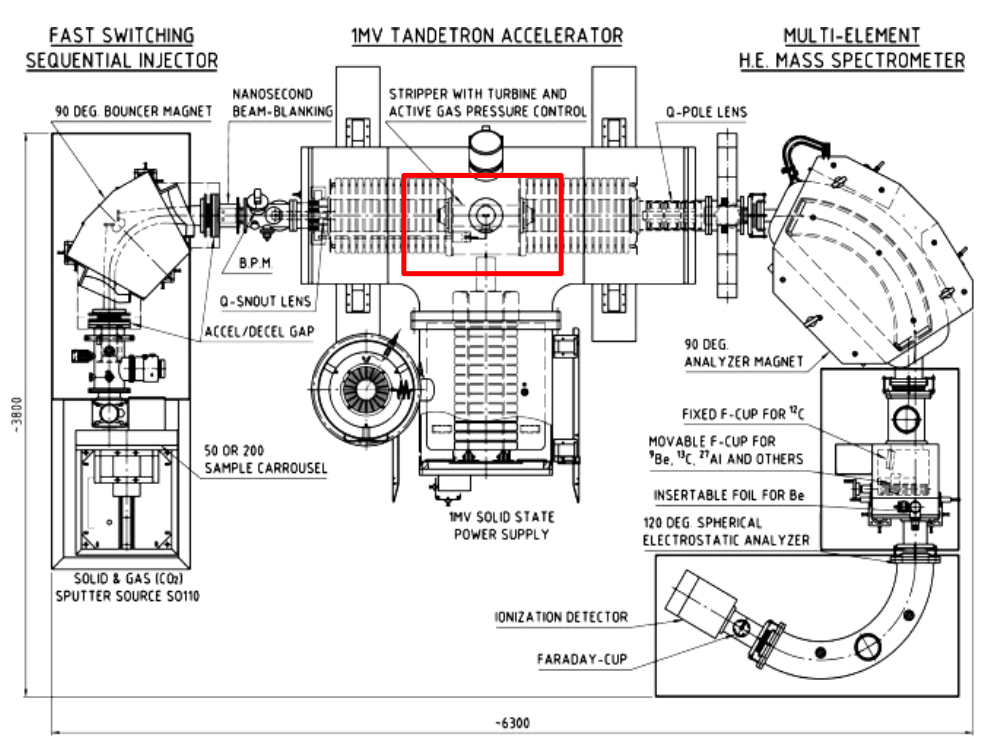




\section{Results (1/2)}

\begin{tabular}{|c|c|c|c|c|c|}
\hline Isotope & $\begin{array}{c}\text { Charge } \\
\text { state }\end{array}$ & $\begin{array}{c}\text { Terminal } \\
\text { voltage } \\
\mathbf{( k V )}\end{array}$ & $\begin{array}{c}\text { Energy } \\
\mathbf{( k e V )}\end{array}$ & $\begin{array}{c}\text { Maximum } \\
\text { transmission } \\
\text { Ar (\%) }\end{array}$ & $\begin{array}{c}\text { Maximum } \\
\text { transmission } \\
\text { He (\%) }\end{array}$ \\
\hline Beryllium & $1+$ & 1000 & 1370 & 58 & 60 \\
\hline Beryllium & $2+$ & 1000 & 2370 & 25 & 23 \\
\hline Aluminum & $2+$ & 1000 & 2650 & 54 & 18 \\
\hline Aluminum & $3+$ & 1000 & 3650 & 13 & 27 \\
\hline lodine & $3+$ & 1000 & 4030 & 10 & 50 \\
\hline Uranium & $3+$ & 650 & 2590 & 13 & 60 \\
\hline
\end{tabular}




\section{Results (2/2)}

\begin{tabular}{|c|c|c|c|c|}
\hline Isotope & $\begin{array}{c}\text { Charge } \\
\text { state }\end{array}$ & $\begin{array}{c}\text { Terminal } \\
\text { voltage } \\
\mathbf{( k V )}\end{array}$ & $\begin{array}{c}\text { Maximum } \\
\text { transmission } \\
\text { Ar (\%) }\end{array}$ & $\begin{array}{c}\text { Maximum } \\
\text { transmission } \\
\text { He (\%) }\end{array}$ \\
\hline Beryllium & $1+$ & 1000 & 58 & 60 \\
\hline Beryllium & $2+$ & 1000 & 25 & 23 \\
\hline
\end{tabular}

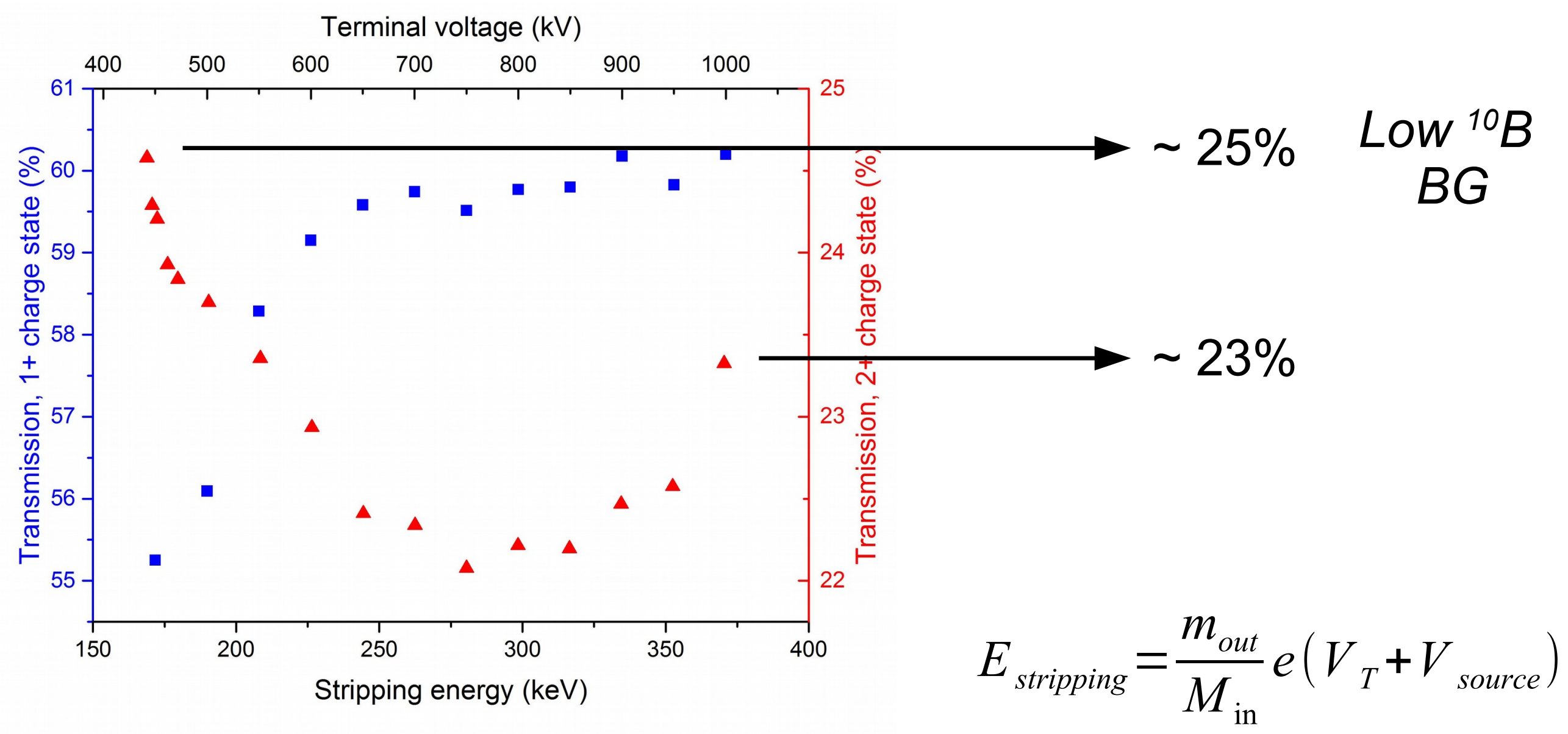




\section{Improving the detection}

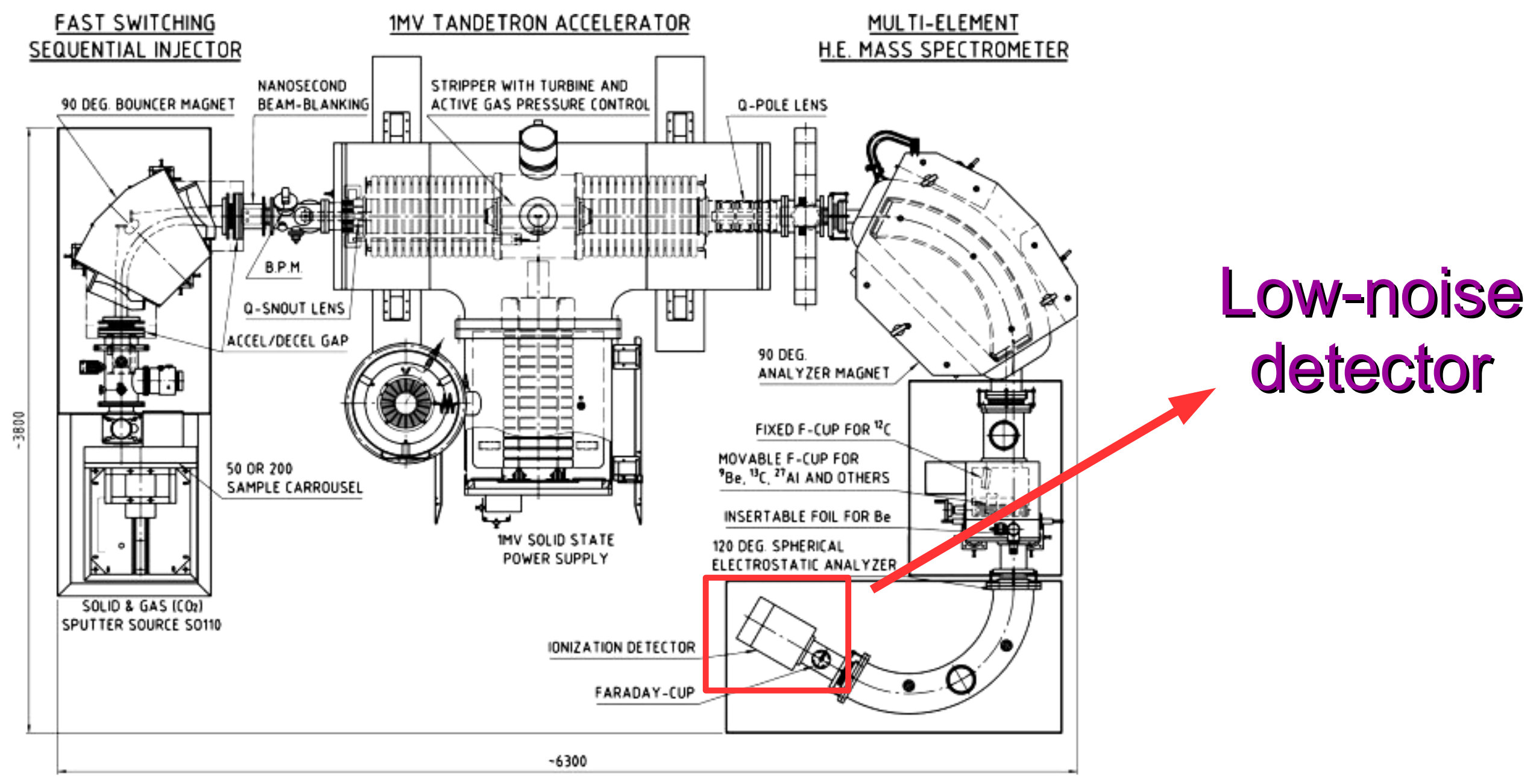




\section{The rare isotope detector}

Gas Ionization Chamber

* Rare isotope

× Isobutane

$\times$ Two anodes $\times$ Frisch grid

× $\mathrm{Si}_{3} \mathrm{~N}_{3.2}$ entrance foil

$\times$ Dedicated electronics

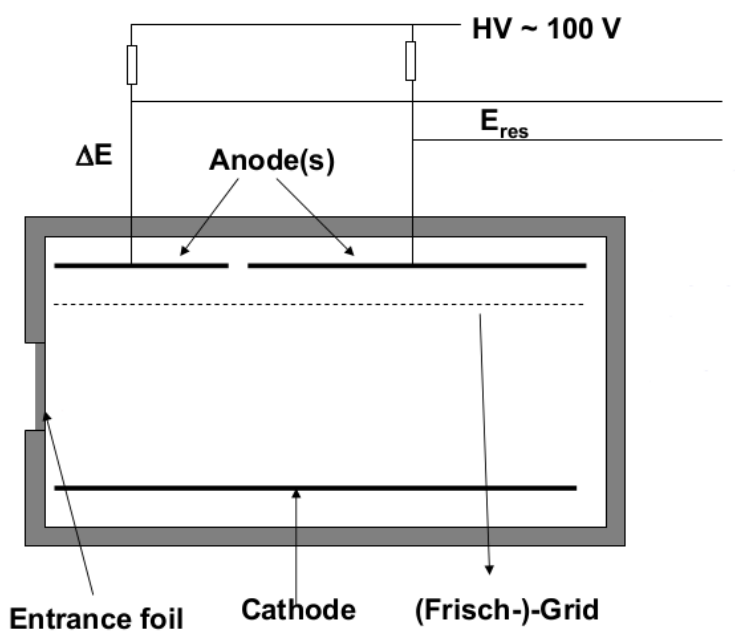

Second anode

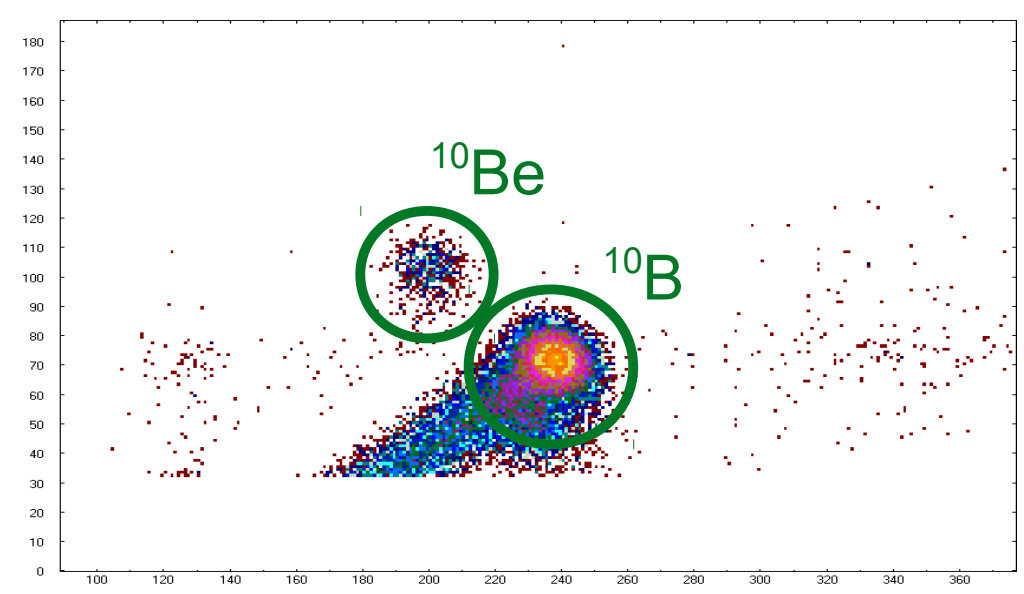

First anode 


\section{Improving the detection}

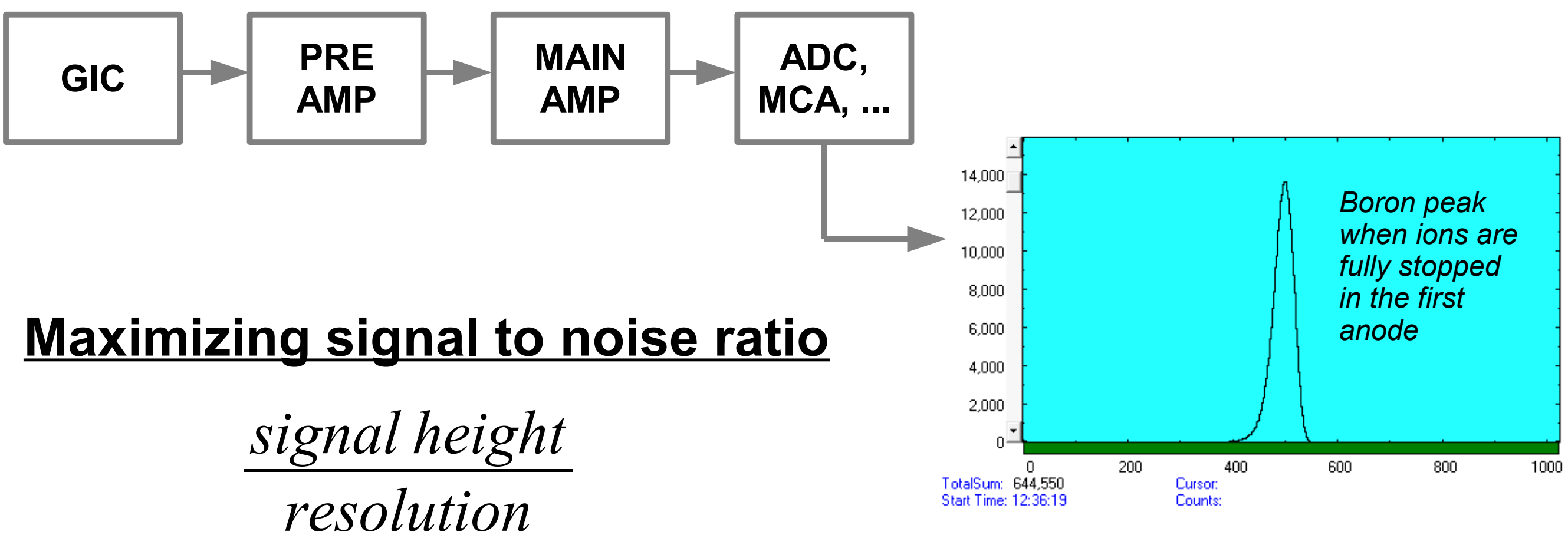

Reduce:

- Geometrical effects

- Energy straggling in the entrance window

- Electronic noise
Increase:

- Ionization yield per energy loss

- Efficiency in charge collection

- Active area 
Very low-noise design

$\rightarrow$ Dead areas minimized

$\rightarrow$ CREMAT preamplifiers

$\rightarrow$ Signal cable length minimized
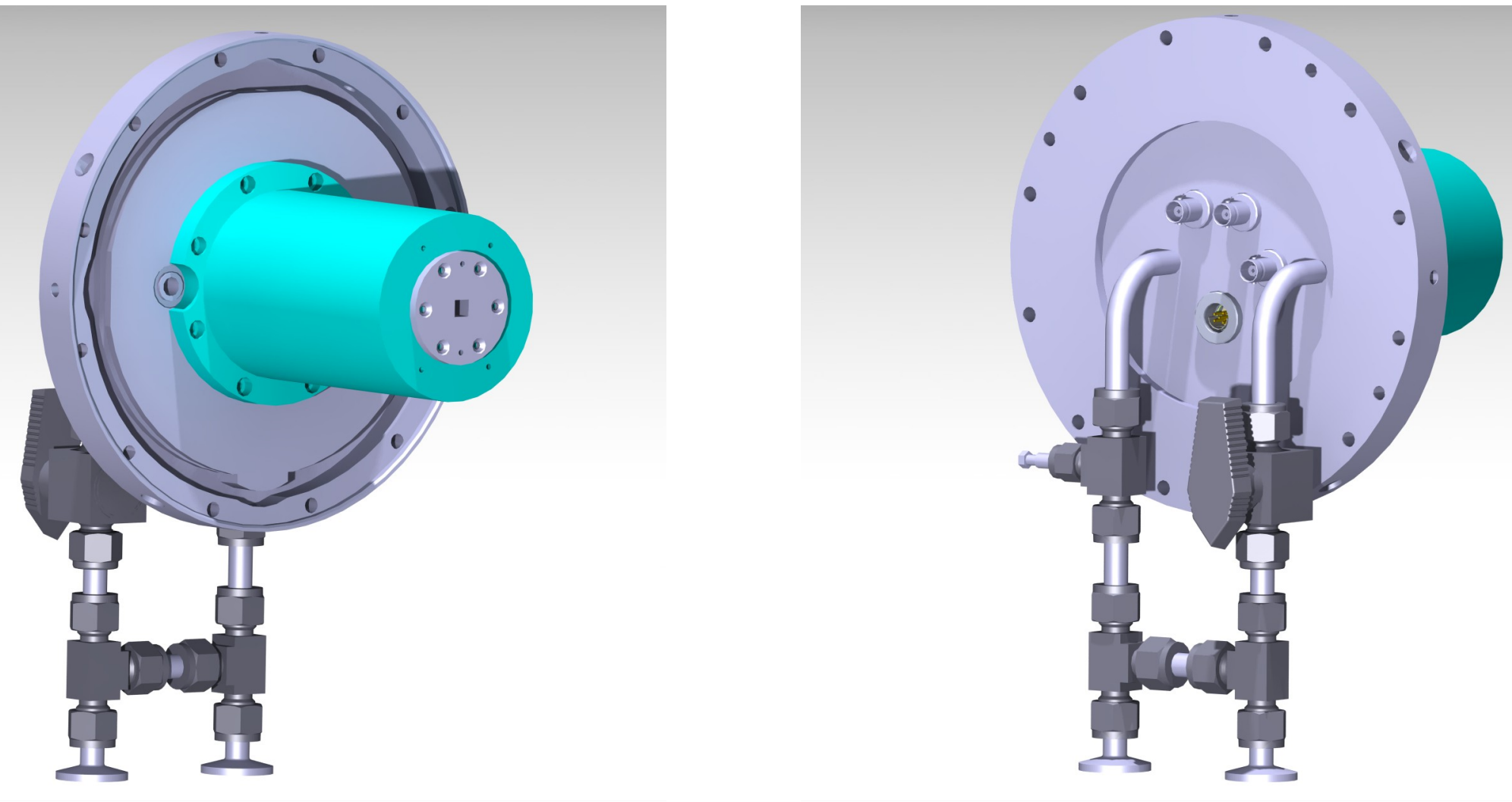

Thank you Dr. Miller! 

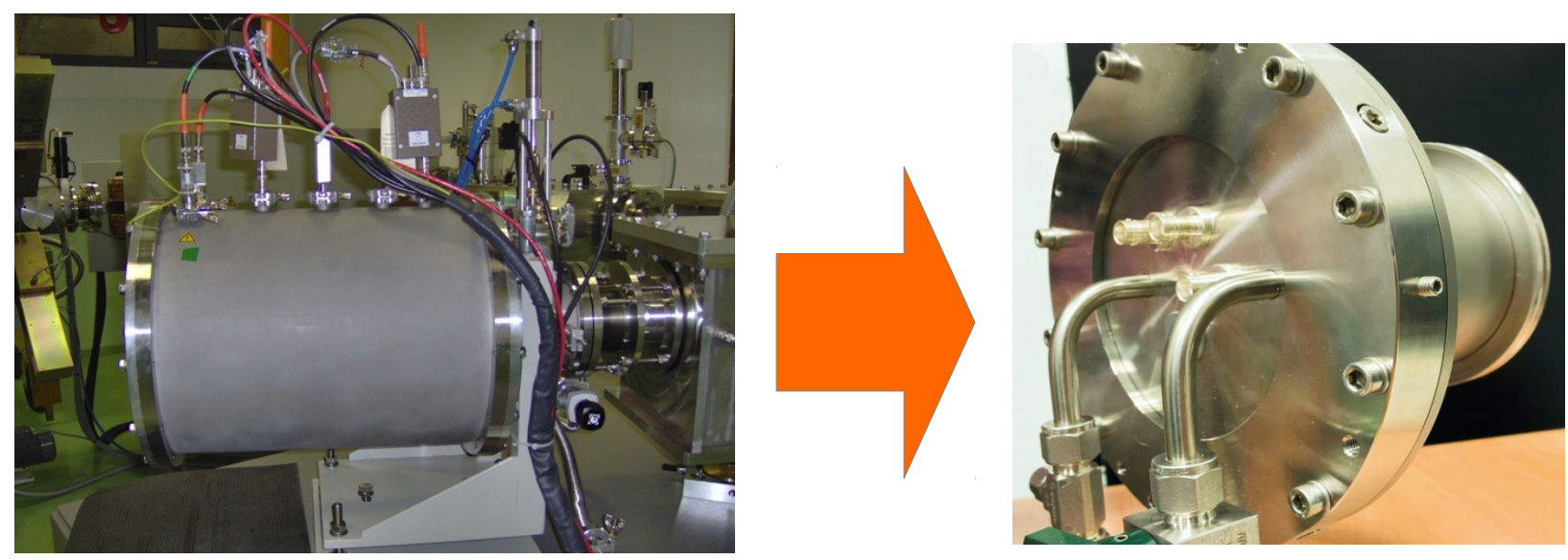

\begin{tabular}{|c|c|c|}
\cline { 2 - 3 } \multicolumn{1}{c|}{} & Originally installed & Current set-up \\
\hline Anodes length & $32 \mathrm{~cm}$ & $10 \mathrm{~cm}$ \\
\hline Preamplifiers & ORTEC & CREMAT \\
\hline Amplifiers & ORTEC & CANBERRA \\
\hline Amplifiers shaping time & $1.5 \mu \mathrm{s}$ & $1.5 \mu \mathrm{s}$ \\
\hline
\end{tabular}




\section{Results}

- Part 1: He stripping

- Part 2: detector

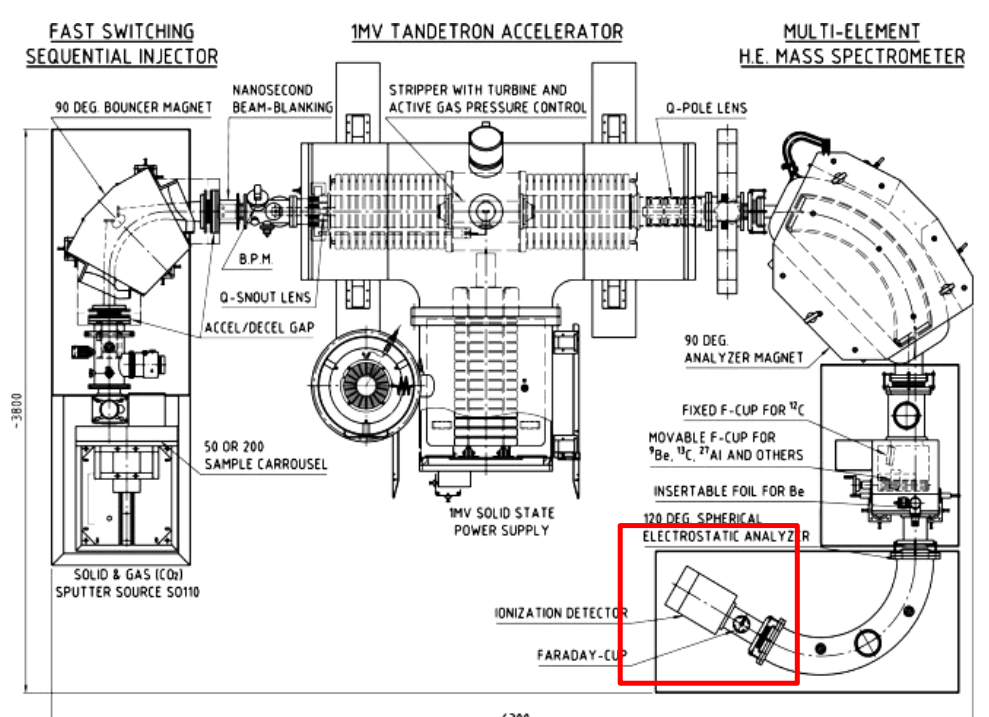




\section{Results}

$\mathbf{R}_{\text {gas }}$ : Charge production yield and collection

$\mathbf{R}_{\text {noise }}$ : Electronic noise (detector, preamplifier)

$\mathbf{R}_{\text {foil }} \quad$ : Straggling due to the SiN entrance window (Sun et al., 2007)

$$
R_{\text {tot }}^{2}=R_{\text {gas }}^{2}+R_{\text {noise }}^{2}+R_{\text {foil }}^{2}
$$

\begin{tabular}{|c|c|c|c|c|c|}
\hline & $\begin{array}{c}\text { Gas } \\
(\mathbf{k e V})\end{array}$ & $\begin{array}{c}\text { Electronic } \\
\text { noise } \\
(\mathbf{k e V})\end{array}$ & $\begin{array}{c}\mathrm{Si}_{3} \mathbf{N}_{3.2} \\
\text { thickness } \\
(\mathbf{n m})\end{array}$ & $\begin{array}{c}\mathrm{Si}_{3} \mathbf{N}_{3.2} \\
\text { straggling } \\
(\mathbf{k e V})\end{array}$ & $\begin{array}{c}\text { Full } \\
\text { resolution } \\
(\mathrm{keV})\end{array}$ \\
\hline Old GIC & 26 & 29 & 40 & 8 & 40 \\
\hline ETH GIC & 25 & 22 & 50 & 9 & 34 \\
\hline
\end{tabular}

For a ${ }^{9} \mathrm{Be}$ beam at $1300 \mathrm{keV}$, with ions fully stopped in the first anode 


\section{What's next?}

Current setup

- Efficiency

- Background

- High counting rates ( $10 \mathrm{kHz}$ )

Useful upgrades

- Passive absorber cell

- TOF detector 


\section{Conclusions}

- SARA is a LE-AMS facility that recently underwent some changes to improve its performance.

- He gas has been introduced to increase the transmission of heavy ions through the accelerator.

- A low-noise GIC has been mounted to obtain better resolutions.

- Useful upgrades could be helpful to further improve measurement conditions. 
¡Gracias!

Any questions? 